daß es ihm durch seine "Grenzentheorie" gelingt, "die Souveränität Gottes so zu begründen, daß sie der Volkssouveränität Raum für eine menschenrechtsfreundliche Gesetzgebung läßt". (S. 315) Auf wenigen Seiten zieht der Verfasser ein Fazit und gibt einen Ausblick (sechster Teil; S. 319-330). Zu Recht stellt er fest, daß Koran und Sunna nicht notwendigerweise ein Hindernis für die Anerkennung der (politischen) Menschenrechte sind, sondern daß entscheidend ist, "wie (kursiv vom Verf.) mit den Quellen umgegangen wird". (S. 322)

Leider kommt der Rezensent nicht umhin, die im Buch verwendete Umschrift arabischer Wörter und Namen, besonders das Fehlen von diakritischen Zeichen, zu kritisieren. Einige Druckfehler (z. B. S. 80-82), Ungenauigkeiten (z.B. S. 119 und 124) und Wiederholungen (z.B. S. 119 und 134) mindern den Wert der interessanten und inhaltsreichen Arbeit nur unwesentlich. All diejenigen, die sich über die Menschenrechtsproblematik in der islamischen Welt informieren wollen, sollten das Buch zur Hand nehmen.

Hans-Georg Ebert

\title{
Rüdiger Lohlker
}

Schari'a und Moderne. Diskussionen über Schwangerschaftsabbruch, Versicherung und Zinsen

Abhandlungen für die Kunde des Morgenlandes, Band LI,3

Kommissionsverlag Franz Steiner, 1996, 156 S., DM 88,--

Der Titel "Schari'a und Moderne" umreißt die zentrale Problematik des islamischen Rechts und der gläubigen Muslime in der heutigen Zeit: Wie läßt sich das überkommene, religiös sanktionierte Recht auf der Basis sakraler Texte in der heutigen Zeit leben? Das Recht ist dabei nur ein Ausschnitt aus einer Kultur im Umbruch, kann aber ein Zugang zu ihr sein. Im Untertitel werden schwierige und praktisch sehr relevante Bereiche herausgegriffen: Versicherung und Zinsen; dem wird der Schwangerschaftsabbruch beigesellt.

Wie der Untertitel erwarten läßt, versammelt der Band drei sehr unterschiedliche Einzelstudien, die durch das Vorwort nur lose verbunden werden (S. 7-11). Ein abschließendes Resümee fehlt ebenso wie ein Stichwortverzeichnis.

Der Titel verspricht viel. In der Einleitung zeigt sich der Verfasser bemüht, diesen Anspruch einzulösen: "Hiermit soll ein Ausschnitt der in islamischen Ländern - insbesondere in Ägypten - im Umlauf befindlichen Normprojektionen wiedergegeben werden, die auch für die Gesetzgebung von Bedeutung sein können" (S. 8). Allerdings werden in der Einleitung sprunghaft verschiedene Probleme sehr kurz angerissen, meist mit den Worten anderer Autoren. Der Verfasser spricht das Problem des islamischen Rechts an, das nach dem Verlust der gesellschaftlichen Voraussetzungen seiner Anwendung das zuvor implizit Vorausgesetzte nun in Rechtsform gießen muß. Er weist zu recht darauf hin, daß der Islam und das islamische Recht nicht monolithisch sind, sondern daß sich in seinem Rahmen divergierende Positionen, 
Diskussionen und Hinweise auf kulturellen Wandel finden lassen (S. 9). Der Einfluß der Moderne sei dabei "nicht nur auf normativer oder formaler Ebene "nachzuweisen, sondern "eher auf inhaltlich-argumentativer bzw. diskursiver Ebene auffindbar" (S. 10). Ebenfalls zu recht nimmt er das islamische Recht gegen (ungenannte) Angriffe auf seinen Rechtscharakter in Schutz.

Im ersten Teil "Zur Abtreibung im islamischen Recht" (S. 13-45) gibt der Verfasser den Inhalt eines Rechtsgutachtens wieder, das der ehemalige ägyptische Mufti und Scheich der al-AzharUniversität im Jahre 1980 erteilt und im Jahre 1987 wiederveröffentlicht hat. Der arabische Originaltext ist mitabgedruckt. Leider erschöpft sich der Abschnitt tatsächlich in einer Wiedergabe des Inhalts dieses Gutachtens. Analyse findet sich nur in Ansätzen. Auch der der "Argumentation Gad al-Haqqs" gewidmete Abschnitt geht kaum über eine Inhaltsangabe hinaus. Die breitere Diskussion wird allenfalls angedeutet und kaum bis in die jüngste Zeit fortgeführt.

Die zweite und längste Studie zu "Versicherung und islamisches Recht" (S. 47-105) enthält "Materialien zur islamischen Diskussion über das Versicherungswesen" (so der Untertitel), die der Autor aus dem Arabischen übersetzt hat, ohne allerdings immer klar zwischen Übersetzung und Kommentar zu trennen. Der Teil beginnt mit einer kurzen Einleitung, in der vor allem ein historischer Überblick über die Diskussion im islamischen Recht gegeben wird. Dem Abschnitt 1.1. folgt dann nicht 1.2. Vielmehr geht der Verfasser gleich zu 2., den "Materialien" über. Hier finden sich zunächst Übersetzungen von Stellungnahmen verschiedener Institutionen, die den kommerziellen Versicherungsvertrag für unzulässig halten und dafür vor allem auf das Verbot von garar (Risiko) und riba (Wucher) verweisen. Als Alternative propagieren sie ein genossenschaftliches Versicherungsmodell. Der Begriff tabarru ${ }^{\mathrm{c}}$ wird mal als "Spende" (S. 61), häufiger jedoch als "einseitig verpflichtendes Geschäft" (z.B. S. 95, 96) oder "einseitig verpflichtender Vertrag" (S. 77) übersetzt. Richtiger wäre es wohl, durchgehend von unentgeltlichen Geschäften zu sprechen, die dem kommerziellen, als Austauschvertrag verstandenen Versicherungsvertrag als Alternative gegenübergestellt werden. Bei unentgeltlichen Geschäften gilt das Risikoverbot nicht.

Nicht alle Institutionen werden vom Verfasser vorgestellt, nicht alle Stellungnahmen werden datiert, nicht für jede wird eine Fundstelle angegeben. Die Übersetzung bedürfte an einigen Stellen noch der Überarbeitung. Manche Sätze geben ihren Sinn auch nach mehrmaliger Lektüre nicht preis (z.B. S. 75: "Zu den Unterscheidungsmerkmalen zählt, daß die Grundlage (asl) dafür, daß der Sippenverband die Zahlung des Wergeldes (diya) bei der Tötung infolge eines Irrtums (hata) bzw. unter bedingtem Vorsatz (sibh al- ${ }^{\mathrm{c}}$ amd) auf sich nimmt, ist das, was zwischen ihr und dem infolge eines Irrtums bzw. unter bedingtem Vorsatz Getöteten an Verwandtschaft besteht, die nach Beistand, Verbundenheit, gegenseitiger Hilfe und dem Erweisen anempfohlener Dinge - selbst ohne Empfangen einer Gegenleistung - verlangt."). Diesen ablehnenden Stellungnahmen wird dann ein längerer Auszug aus einem Werk von Mustafa azZarqa' gegenübergestellt, der sich mit islamisch-rechtlicher Argumentation für die Zulässigkeit des Versicherungsvertrags ausspricht. Im Zentrum seiner Ausführungen steht das Argument, daß neue Vertragstypen - wie der Versicherungsvertrag - im islamischen Recht dann aner- 
kannt werden könnten, wenn die Bedürfnisse der Praxis das erforderten. Dieser Teil gehört zu den interessantesten des Buches, weil hier tatsächlich ein Ausschnitt aus einer relativ aktuellen Diskussion gegeben wird. Er wird abgeschlossen durch einen Abschnitt über "Islamisches Recht und Moderne", der seiner Überschrift allerdings nicht ganz gerecht wird. Der Verfasser erklärt die gerade dargestellten Meinungen mit einem Zitat als zwei unterschiedliche Wege "ideologischer Rechtfertigung der Integration westlicher Werte und Normen und, ökonomisch gesehen, kapitalistischer Strukturen in den islamischen Gesellschaften" (S. 104). Den Prozeß der Fortentwicklung der überlieferten religiösen/rechtlichen Gedankenwelt gelte es "in detaillierter, empirischer Untersuchung zu rekonstruieren" - ein äußerst lobenswerter Vorsatz für die Zukunft.

Der dritte Teil (S. 107-141) ist der Frage "Sind Zinsen im Islam erlaubt?" gewidmet. Die kurze Einleitung enthält auch Ausführungen "zum Begriff des Zinses im bundesdeutschen Recht", die allerdings sehr verschwommen bleiben und zum Verständnis des Folgenden nichts beitragen. Interessanter wäre eine kurze Darstellung der aktuellen ägyptischen Rechtslage gewesen. Im Hauptteil gibt der Verfasser den Inhalt eines Werkes wieder, in dem der hohe ägyptische Richter al-Asmawi sich für eine eingeschränkte Zulässigkeit von Zinsen ausspricht. Als Kontrast dazu werden im Kursivdruck immer wieder einzelne Argumente aus der Replik eines Gelehrten der al-Azhar (al-Hatib) wiedergegeben, der das Zinsverbot verteidigt. Diese Methode ist deshalb interessant, weil sie es ermöglicht, zwei repräsentative Strömungen in dieser Frage unmittelbar nebeneinander zu verfolgen: Al-Asmawi spricht sich für eine "Kontextualisierung" der "heiligen" Texte und eine Reinterpretatition des Rechts im Lichte der Moderne aus. Al-Hatib vertritt die Position des traditionellen islamischen Rechts. Auf diese Weise wird das Dilemma des islamischen Rechts deutlich, das einerseits auf seine religiöse Basis nicht verzichten kann, das jedoch andererseits in vieler Hinsicht den Anforderungen der Gegenwart nicht gerecht wird. In der "Schlussbemerkung" zu diesem Teil werden noch einmal kurz die Unterschiede der Argumentation angesprochen, ansonsten bleibt sie jedoch recht blaß.

Die hochgesteckten Erwartungen, die der Titel weckt, kann das Werk nicht erfüllen. Es bietet einige interessante Momentaufnahmen und kann den Leser an manchen Stellen an den Bemühungen islamischer Juristen teilhaben lassen, eine adäquate Antwort auf Probleme der Moderne auf dem Boden des islamischen Rechts zu geben. Die einzelnen Teile sind jedoch sehr uneinheitlich, die Auswahl wirkt etwas zufällig und vermittelt den Eindruck einer persönlichen Materialsammlung, die den Nachweis der Repräsentativität schuldig bleibt. Hinweise auf das geltende Recht fehlen weitgehend. Die analytischen Passagen sind sehr kurz und enthalten ungemein viele wörtliche Zitate, ein Gesamtresümee wird nicht gezogen. Die Übersetzungen sind stilistisch noch verbesserungsbedürftig und vor allem im zweiten Teil häufen sich die Fehler in der Rechtschreibung und im Satzbau. Insgesamt macht das Buch einen unfertigen Eindruck, es wirkt eher wie eine interne Zwischenstufe auf dem Weg zu weiteren Forschungen - die allerdings versprechen interessant zu werden.

J. Christian Wichard 